\title{
Automated classification of peripheral distal by-pass geometries reconstructed from medical data
}

\author{
S. Giordana, S. J. Sherwin ${ }^{1}$, J. Peiró, and D. J. Doorly, \\ Biofluids Group, Department of Aeronautics, Imperial College London, SW7 2BY, UK \\ Y. Papaharilaou, C. G. Caro and N. Watkins \\ Bioengineering Department, Imperial College London, U.K. \\ N. Cheshire, M. Jackson, C. Bicknall and V. Zervas \\ Regional Vascular Unit, Saint Mary's Hospital, Imperial College London, U.K.
}

\begin{abstract}
Abnormal haemodynamic conditions are implicated in the development of anastomotic myointimal hyperplasia (MIH). However these conditions are difficult to determine in-vivo, prompting research using ex-vivo idealised models. To relate the understanding gained in idealised geometries to anatomically correct conditions we have investigated a reproducible approach to classify in-vivo distal graft anastomoses and their inter-patient variability.

In-vivo distal anastomotic geometries were acquired by magnetic resonance (MR) angiography from 13 patients who had undergone infrageniculate autologous venous bypass surgery. On average, the images were acquired 2 weeks post-operatively. Five patients also underwent repeat examinations 2 to 7 weeks later. For each geometry, the surface of the arterial lumen is represented by the zero level set of an implicit function constructed from radial basis functions that minimize curvature.

The three-dimensional binary image created from the interpolated surface is processed using a skeletonisation algorithm to obtain the centreline of each branch in the geometry. This allows for the measurement of the branching angles between straight line approximations of the centrelines of each vessel, averaging them over a characteristic length of each anastomosis.

The main finding in the application of the proposed classification methodology to this set of patients is that the spectrum of anastomoses can be reduced to a small subset of cases characterized by two angles: the angle between the graft and the plane of the host artery and the angle between the graft and the proximal branch of the artery.
\end{abstract}

Keywords: anastomosis, reconstruction, implicit functions, geometry.

\section{Introduction}

In this work we attempt a systematic classification of the geometry of the distal anastomosis of end-to-side peripheral by-pass grafts. The ultimate aim of the classification is the assessment of the variability of surgically constructed anastomoses. Issues are the reproducibility of some configurations, but also the capability to follow the evolution of the shape of a distal anastomosis post-operatively.

The tools presented in this paper permit an automated three-dimensional geometrical reconstruction from MR images and require minimum user intervention. This geometric definition is required for the generation of computational fluid dynamics (CFD) grids [16]. Understanding the geometrical variability of the acquired anastomosis is the first step towards the investigation of relations that may exist between shape and haemodynamic factors that CFD can compute such

\footnotetext{
${ }^{1}$ Author for correspondence
} 
as wall shear stresses and particle residence times. The ability to identify geometrical parameters that influence the haemodynamic behaviour within an anastomosis may eventually lead to a more thorough understanding of the performance of peripheral by-pass grafts.

Many techniques have been proposed to address the reconstruction of a three-dimensional shape from medical images. All the reconstruction methods need some image processing tool, such as edge detection algorithms or grey-scale thresholding. Once images have been processed, a final surface needs to be generated and probably smoothed. The main distinction between the techniques is the final surface representation, which may be given in terms of a triangulation or as a set of connected parametric surface patches.

The work of Long et al. [12] describes the extraction of contours of the lumen of the carotid bifurcation and the generation of a parametric surface, stressing the importance of smoothing not only the contours but also their position along the axis of each vessel, which is the line formed by the centroid of the contours. The same work does not specify how the surface interpolation handles the change of topology that afflicts branching vessels. A similar reconstruction technique is also proposed by Moore et al. [14], with the same lack of description about how to fit a set Bspline patches around an anatomically correct carotid bifurcation, specifically ignoring the detail of the change in topology. Another use of B-spline patches to be fitted along planar contours is presented in Perktold et al. [17] and applied to peripheral by-pass grafts in Leuprecht et al. [11]. The authors of these two works explicitly discuss the assumptions they need to treat the by-pass anastomosis, where the topology of the vessels changes in the same way as it does for a carotid bifurcation. The work of Moore et al. [13, 14] addresses the effect of imperfections in the reconstructions on the results of CFD computations and supports the need of smoothing the reconstructed surface in order to eliminate artifacts typically found in the MRI data. As a final remark on these reconstruction methods that produce a surface representation as a set of connected B-spline patches, it is relevant to state that fitting patches through contours of a bifurcation can be quite laborious and needs some assumptions on the topology change that is an intrinsic characteristic of an end-to-side anastomosis.

Cebral and Löhner [4] propose a reconstruction method that delivers a final surface described in terms of a triangulation. Their work does not make use of planar contour extraction algorithms but all the operations are performed directly in 3D space but for image filtering. Images are stacked to form a three dimensional array of voxels where a region growing algorithm assigns to a region of interest all the voxels belonging to a user-selected vessel or group of vessels. A surface is then generated by tessellating the voxels that are on the external bound of the region of interest. Triangulation smoothing algorithms such as that described in [20] provide the final reconstructed surface.

This paper proposes a reconstruction method that represents the shape of a blood vessel as the zero-level set of an implicit function fitted through the contours obtained by thresholding medical images. The resulting surface is generated by triangulating an isosurface of a scalar field. Properties of the implicit function fitting reside in an intrinsic capability to deal with changes in topology together with the assurance of getting a smooth surface once an appropriate implicit function is adopted [21]. In two dimensions, for an appropriate choice of basis functions, this technique is equivalent to a spline fit through a discrete set of points. The greatest advantage of this method is the minimum user intervention, which is limited to the thresholding part of the procedure. The isosurface of the implicit function may require further smoothing when the purpose of the reconstruction is the generation of CFD meshes, which this paper does not discuss. On the other hand, it will be shown that further smoothing is not required when a reconstruction is intended for shape classification purposes only.

Most of the operations involved in the reconstruction of the geometry of the vessel from medical data and the extraction of features are well documented procedures in computer graphics applications. Many of the algorithms presented in this work have been borrowed from the extensive literature in the subject. However, the degree of geometric fidelity required for flow modelling using CFD is much higher than that required for visualization in computer graphics and animation. For example, a maximum intensity projection shown in figure 1(b) is an established visualisation technique that provides surgeons with sufficient three-dimensional information from MR images. 


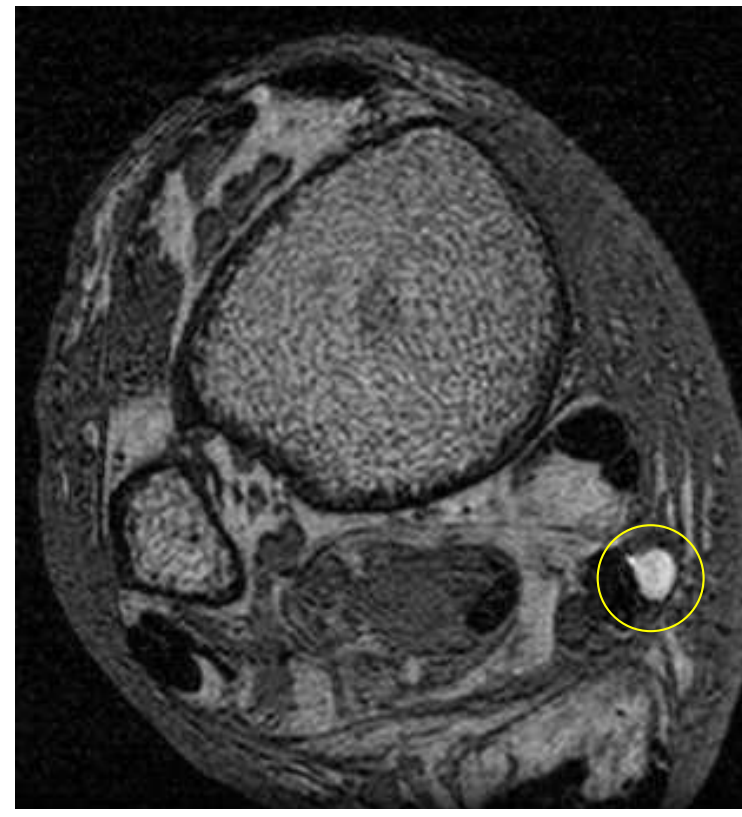

(a)

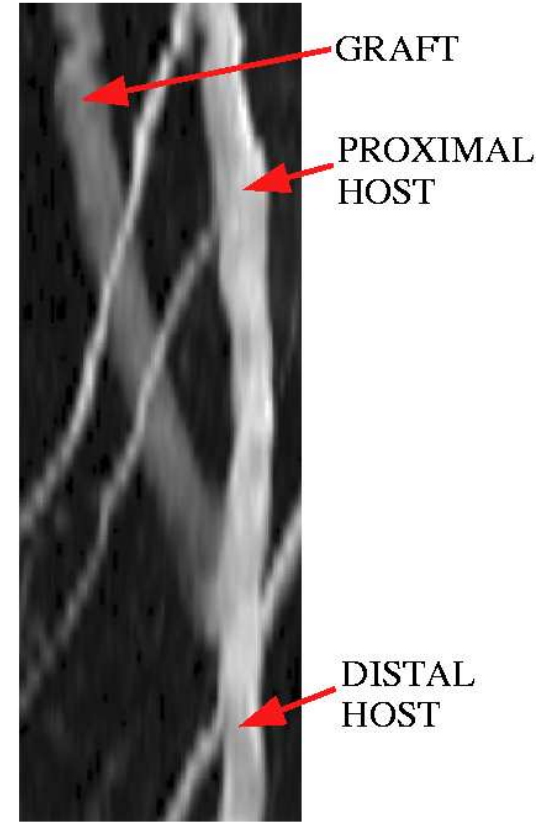

(b)

Figure 1: (a) Raw MRI image: The blood flow region is visible as a brighter white area within the circle superimposed to the grayscale image. (b) Maximum intensity projection showing a three-dimensional visualisation of an anastomosis and the surrounding blood vessels.

Still, the results of such a projection do not constitute a geometric reconstruction suitable for CFD mesh generation or geometry classification. This means that, in practice, only a small set of the available algorithms are appropriate for the reconstruction of geometries for CFD modelling. The main contribution of the research presented here has been the selection of suitable algorithms for geometry reconstruction and feature extraction and their modification to achieve the levels of geometric fidelity required for accurate CFD modelling such as that offered by high-order spectral/hp Navier-Stokes solvers $[9,16]$.

This paper is organised as follows. In section 2 we discuss the slice acquisition and reconstruction of the lumen using spline fitting within the data slices and a surface interpolation by implicit functions. In section 3 we discuss the accuracy of the reconstruction process by considering some well defined model problems. In section 4 we introduce a centreline extraction technique using a skeletonization algorithm. We then discuss the evaluation of the cross sectional area in section 5 which is used to define a characteristic scale for the reconstruction. Finally in section 6 we define the parameterisation of the bifurcation based on the extracted centrelines and apply it to a study of twenty in-vivo peripheral bypass grafts.

\section{Slice data acquisition and extraction of the lumen}

The imaging technique adopted here provides a set of two-dimensional pictures formed by $512 \times 512$ pixels with their intensity represented on a scale of 256 levels of grey. Specifically the MRI scans were obtained from a whole body General Electric 1.5 Tesla scanner with peak gradient strength of $20 \mathrm{mT} / \mathrm{m}$. Images were acquired by MR Angiography pulse sequence based on axial 3D vascular time of flight. The parameters of the acquisitions are: Field of View 12cm, phase FOV 0.8, spatial resolution $256 \times 256$ interpolated to $512 \times 512$ by K-space zero filling, TE $3.5 \mathrm{~ms}$, TR $47 \mathrm{~ms}$, and a slice thickness of $1 \mathrm{~mm}$. Regions of blood flow are identified by a brighter intensity than the 


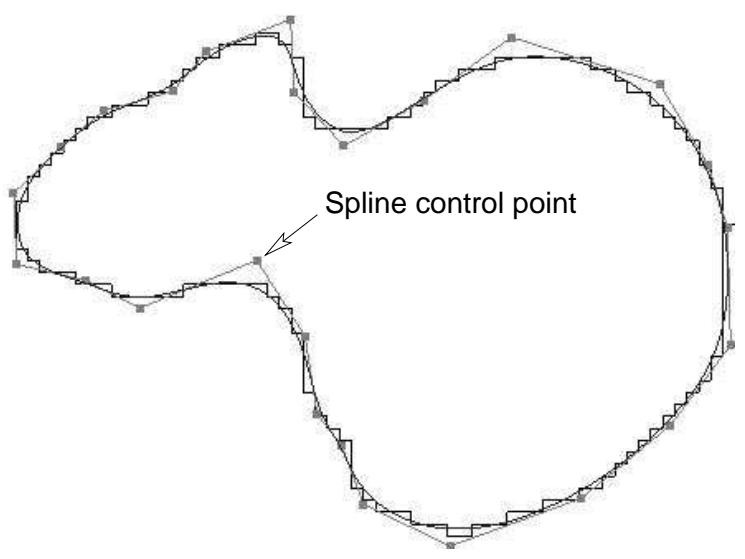

Figure 2: Segmented line and spline curve approximation: spline control points are shown.

surrounding tissue as illustrated in figure 1. Segmentation was then carried out using a freely available computer code ${ }^{2}$ that implements standard edge detection and thresholding techniques to provide a pixelated boundary of the image. Choosing a region of interest, only those pixels that are brighter than a given threshold level are segregated from the background. On each MRI slice the region of interest is then bounded by a closed segmented line representing the arterial lumen and shaped like a staircase as depicted in figure 2 .

The process yields a set of planar closed polygonal lines, each of them bounding the arterial lumen at a different longitudinal coordinate value. Since the segmentation is the first step in the manipulation of the images, it is legitimate to question the amount of error it introduces in the data. As a base line assumption we consider the MRI scanner "error free". It is hard to estimate the segmentation accuracy as a certain amount of user intervention is required to eliminate unwanted branching vessels or to take particular care in areas of flow artifacts. On the other hand, the best resolution that the segmentation can achieve is one pixel of the image. The imaging technique used in this work yields about 20 pixels along the diameter of grafts (usually around $5 \mathrm{~mm}$ ), while the resolution can be as low as only 5 pixels along the diameter of the host vessels in some stenosed cases. It follows that, in the worst case, adding or removing one single pixel from a contour could result in errors in the diameter of the vessel section of $20 \%$ or more and will introduce significant errors in the reconstruction.

\subsection{Spline fitting of the planar profiles}

In the next step of the surface reconstruction, each of the planar profiles is smoothed and represented in a parametric form. To achieve this, we employ cubic splines that approximate each curve in a least-squares sense. The accuracy of the spline fitting process is therefore linked to the degree of smoothing applied. As a general rule, the maximum allowable smoothing was restricted so that the approximating curves would not differ more than one pixel from the segmented lines.

\subsection{Surface interpolation using an implicit function}

The spline representation of the 2D slices is evaluated at a fixed number of points that we will refer to as the surface constraints. The planar normal to a curve within a slice is also computed at the same points. A second set of points is generated by displacing each of the lumen points along its associated normal towards the inside of the lumen by a distance of 0.1 pixels. We call these the normal constraints. Following Turk and O'Brien [21], the surface is represented as a level set of an unknown implicit function $f(\mathbf{x})$, where $\mathbf{x}=\left(x_{1}, x_{2}, x_{3}\right)$ denotes a point in Cartesian

\footnotetext{
${ }^{2}$ www.scioncorp.com
} 


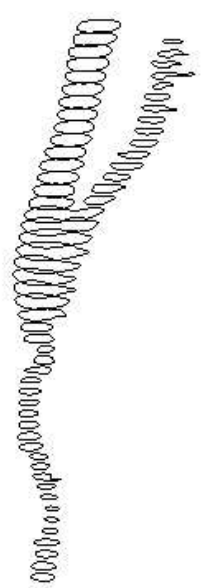

(a)

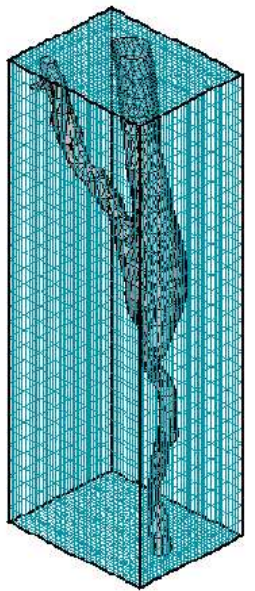

(b)

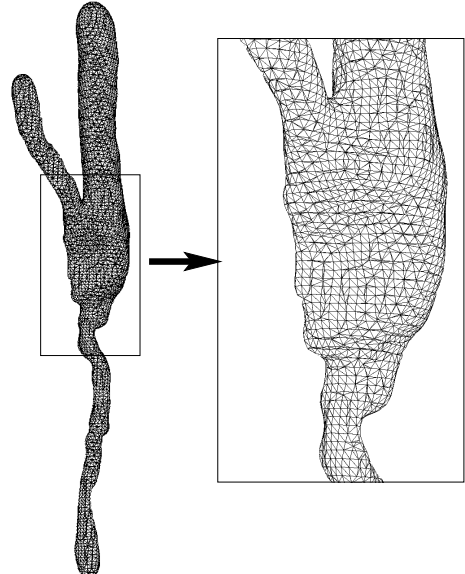

(c)

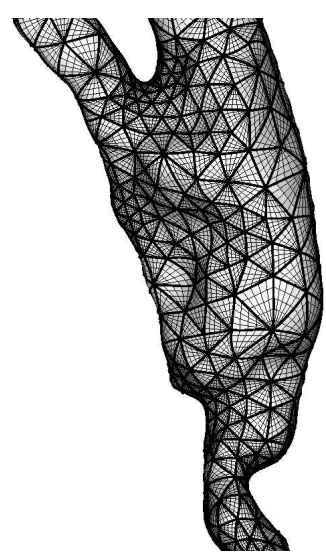

(d)

Figure 3: Interpolation of a set of MRI images: (a) interpolated slice data, (b) a shaded view of the isosurface corresponding to the zero value of the implicit function, (c) surface triangulation generated by Bloomenthal surface polygoniser and (d) high-order surface mesh suitable for CFD.

coordinates. This function is given a zero value on the surface constraints and a unit value on the normal constraints. In the present context, the arterial lumen is the set of points where $f(\mathbf{x})=0$, while any point inside the lumen is characterised by a positive value of $f$.

The surface fitting problem then becomes that of seeking a function $f$ that smoothly goes through these two sets of constraints. The interpolating function $f$ is defined as

$$
f(\mathbf{x})=\sum_{i=1}^{n} c_{i} \phi\left(\mathbf{x}-\mathbf{x}_{i}\right)
$$

where $\phi(\mathbf{x})$ is a radial basis function and $\mathbf{x}_{i}$ are the interpolation constraints.

Given a family of radial basis functions $\phi(\mathbf{x})$, the calculation of the coefficients $c_{i}$ requires the solution of a linear system that results from prescribing the value $h_{i}$ that $f$ attains at the constraint $\mathbf{x}_{i}$. The system is written as

$$
\mathbf{A c}=\mathbf{h}
$$

with

$$
A_{i j}=\phi\left(\mathbf{x}_{i}-\mathbf{x}_{j}\right)
$$

where $h_{i}=0$ if $\mathbf{x}_{i}$ is a surface constraint and $h_{i}=1$ if $\mathbf{x}_{i}$ is a normal constraint.

Radial basis functions suitable for smooth interpolation were originally derived by Duchon [6] as an extension of the "thin plate" functions and are given by $\phi(\mathbf{x})=\|\mathbf{x}\|^{3}$ so that $f$ becomes:

$$
f(\mathbf{x})=\sum_{i=1}^{n} c_{i}\left\|\mathbf{x}-\mathbf{x}_{i}\right\|^{3} .
$$

This function is the multidimensional equivalent of cubic splines that are known to interpolate a set of points while minimising curvature. Computing the coefficients $c_{i}$ in (4), requires solving a semi-positively defined linear system which can be done with an LU factorisation. Further details of the implementation are also given in [16].

\section{$2.3 \quad$ Isosurface extraction}

The zero level set of the function $f$ obtained from the solution of the system (2) is the surface that smoothly interpolates the slices. The extraction of this surface can be accomplished by a marching 


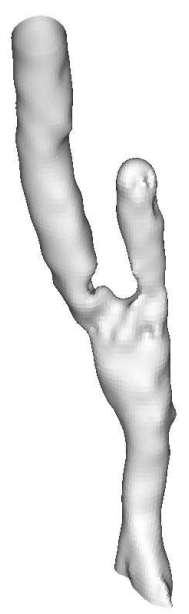

(a)

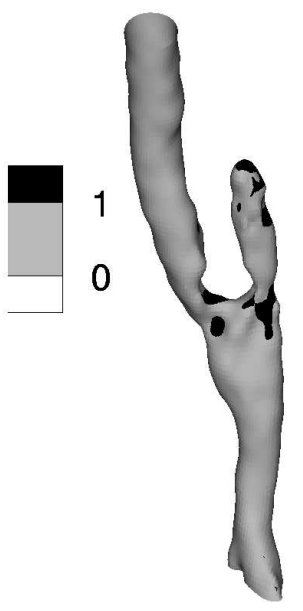

(b)

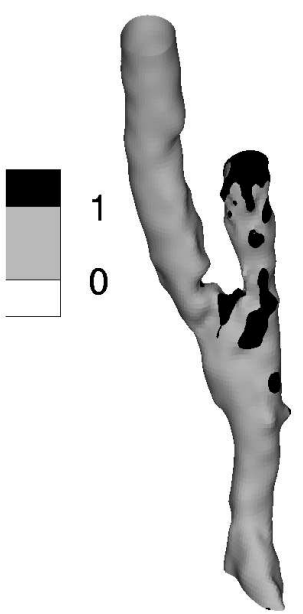

(c)

Figure 4: Effect of thresholding on a graft: (a) shaded surface reconstructed with a threshold of 191. (b) Contours of the distance in pixels between the surface in (a) and a reconstruction with threshold 172, i.e. $10 \%$ lower than 191. (c) Contours of the distance in pixels between the surface in (a) and a reconstruction with threshold 210, i.e. 10\% higher than 191. Areas shaded in dark in (b) and (c) are farther than one pixel from the surface depicted in (a). Most of the differences between the three reconstructions are concentrated at the junction of the graft with the proximal host vessel.

cubes algorithm, either in a simple implementation that requires the repeated evaluation of $f$ over a structured grid, as shown in figure 3(b) or by means of a continuation algorithm presented by Bloomenthal [3] whose resulting triangulation is illustrated in figure 3(c). The latter minimizes the number of evaluations of $f$, thus reducing the computational time.

Both methods produce a triangulation, shown in figure 3, which is adequate for computer graphics purposes but needs further processing to achieve a surface mesh that is suitable for CFD calculations as depicted in figure 3(d). The procedure followed to generate such CFD meshes is detailed in [16] and in [18].

\section{$3 \quad$ Accuracy and fidelity of the reconstruction process}

Taking the MRI images as our starting point, the outlined reconstruction technique produces surfaces that must differ from the original data in three respects:

1. The image segmentation by automatic thresholding requires the user to select a threshold value: the level of gray chosen to represent the boundary of a vessel. The final shape of the lumen is a function of the chosen value and different threshold values lead to different final shapes as shown in figure 4 .

2. Contour fitting by least-squares splines alters the data definition. Errors introduced at this stage are bounded by choosing a smoothing factor that forces the smoothed contours to be closer than one pixel to the original data.

3. The missing information about the surface normals results in an error since the normal constraints are aligned within the plane of the data, not with the normal to the surface. This inevitably leads to the appearance of undulations in the interpolated surface as a result of the lack of out-of-plane information. 


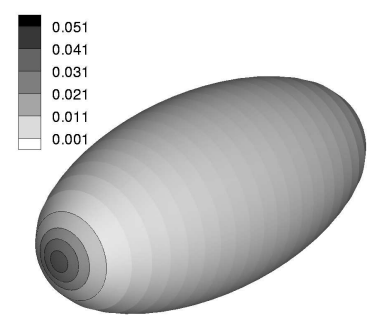

(a)

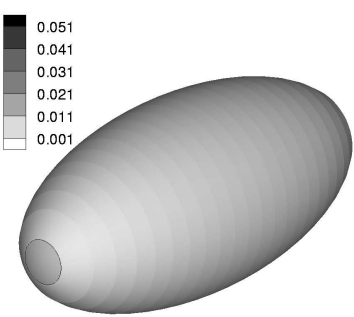

(b)

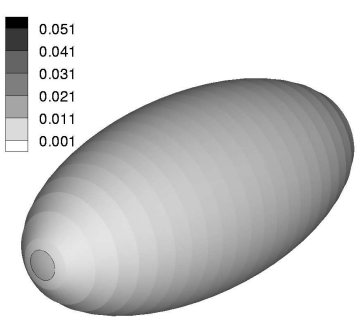

(c)

Figure 5: Assessment of the accuracy of the reconstruction procedure represented by the contours of the distance between an ellipsoid and its reconstruction through a set of slices defined by means of 20 points, normalised by the minor semi-axis: (a) using 10 slices and the sectional normals, i.e. perpendicular to the major axis, (b)using sectional normals and 20 slices, and (c) using the true normal to the ellipsoid and 20 slices.

A quantification of the magnitude of the undulations can be accomplished by reconstructing a known surface using the above described technique. An ellipsoid is sliced into circles evaluated at 20 points and normal constraints are prescribed in the plane of each cross section, for a total of 40 constraints per slice. The reconstructed surface is then projected on the exact ellipsoid and the distance between the two surfaces is computed. Figure 5 shows a distance map: the error in terms of distance to the exact surface, normalised by the minor semi-axis, is of the order of $10^{-4}$ where the surface is flatter, and of about $10^{-2}$ where the curvature is higher, i.e. close to the apex of the ellipsoid.

Furthermore, an investigation of the global reconstruction accuracy must take into account the MRI scanner errors too. If a known shape is imaged and reconstructed, comparing the reconstructed geometry with that of the original object is the easiest way to assess the accuracy of the reconstruction procedure. The research into these issues has started only recently, see for instance [14], and has been mainly motivated by the realisation of the important role that changes in vessel geometry have in relevant haemodynamical quantities such as wall shear stresses [19].

The model used in this work is made up of two tubes joined at an angle of 45 degrees. The tubes were scanned using MRI across 25 planes getting about 16 pixels along the diameter of a slice. The MRI images were segmented using a threshold value of 200. Splines were fitted through each of the 34 cross sections and a set of 40 points were generated on each spline producing a total of 1360 points. The 2720 constraints, of which 1360 are normal constraints, were interpolated using the implicit function approach described previously and the reconstructed model is shown in figure 6(a). The reconstruction shows some artifacts that depend on the MRI scanner, for instance a large indentation can be observed near the junction. This seems to be due to a magnetic susceptibility artifact caused by the presence of the glue used to join the two tubes together. The undulations that are visible on the lateral surface of the tubes are the result of an error in the relative position of the various slices.

Errors in the MRI data and errors introduced by the reconstruction process can be considered as noise that contributes to the final signal. In other words, values of the implicit function $f$ can be seen as a signal affected by noise. A more accurate geometry reconstruction can be achieved by increasing the signal to noise ratio, i.e. by filtering $f$ through a convolution with a Gaussian kernel. This is accomplished by first evaluating $f$ on a structured three-dimensional mesh with $\mathrm{N}^{3}$ points $\mathbf{x}_{i}, i=1, \ldots, \mathrm{N}^{3}$. Then each value $f_{i}$ is averaged with its neighbours along the directions of the three Cartesian axes in sequence. A Gaussian function, with a zero mean, along the $x$-axis and centred at the $i$-th node is given by

$$
K(x ; \sigma)=\frac{1}{\sigma \sqrt{2 \pi}} e^{-\frac{1}{2}\left(\frac{x-x_{i}}{\sigma}\right)^{2}}
$$




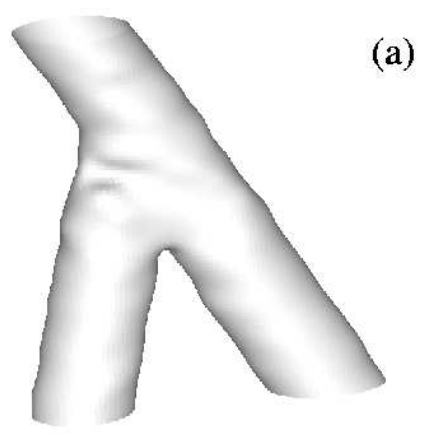

(a)
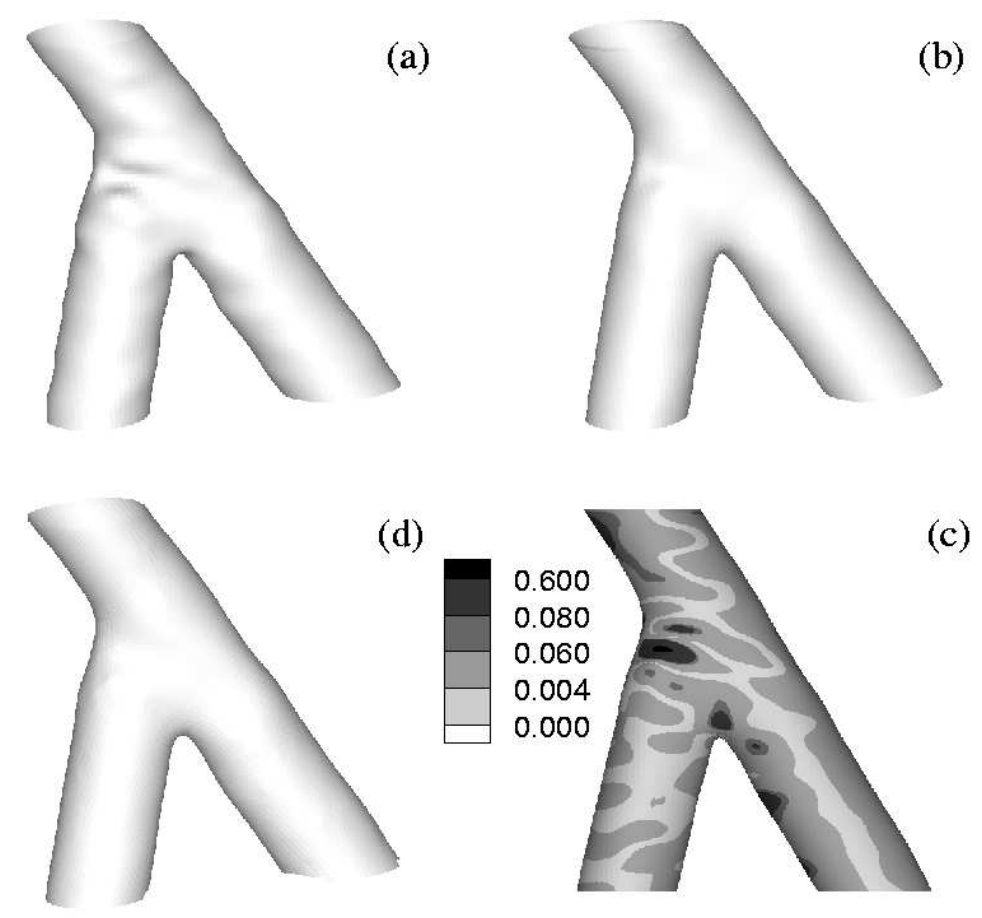

(d)

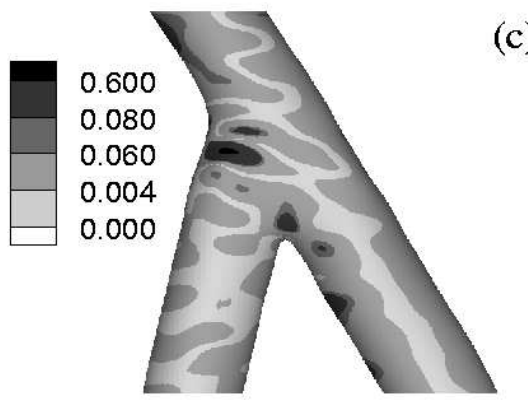

(c)

Figure 6: Reconstruction of a model of two joining tubes: (a) original reconstruction showing the geometric noise, (b) geometry smoothed using the Gaussian convolution filter, (c) distance, expressed in pixels, between the original reconstruction and the one obtained using the Gaussian filter, and (d) geometry smoothed using the low-pass filter.

where $\sigma$ represents the standard deviation of the Gaussian kernel. A similar expression is used for the $y$ and $z$ axes.

In signal processing Gaussian kernels are characterised by the FWHM value defined as the full width of the kernel at half of the maximum of the height of the Gaussian kernel. The relation between the FWHM and $\sigma$ is

$$
\mathrm{FWHM}=\sigma \sqrt{8 \log _{e} 2} .
$$

After defining FWHM, a discrete approximation of the kernel $K$ along the $x$ axis is evaluated at each $x_{i}$. In principle, the Gaussian distribution is non-zero everywhere, but practically it can be forced to a value of zero when at more than three standard deviations from $x_{i}$. Therefore, the kernel can be truncated at $x_{i-l}$ and at $x_{i+l}$ when $\left\|x_{i \pm l}-x_{i}\right\|>3 \sigma$. The discrete kernel $K$ is defined at each $x_{i}$ and at its $2 l$ neighbours, if they exist, giving $2 l+1$ values $K_{i-l}, \ldots, K_{i}, \ldots ., K_{i+l}$, which are obtained by evaluating $K(x ; \sigma)$ and dividing by the area subtended by the kernel. The new smoothed value $f_{i}^{s}$ of $f_{i}$ at $x_{i}$ is then given by

$$
f_{i}^{s}=\sum_{j=i-l}^{i+l} f_{j} K_{j} .
$$

The choice of FWHM is accomplished by a trial and error process. Given a MRI scanner's resolution of one pixel, the maximum allowable smoothing generates a reconstructed surface that must be everywhere closer than one pixel to the original unsmoothed reconstruction. Smoothing a reconstruction with a few different choices of FWHM and computing its distance from the original surface gives distance maps that are useful in deciding the optimum smoothed surface. A smooth surface is shown in figure 6(b) and its distance map from the original reconstruction is shown in figure 6(c). 

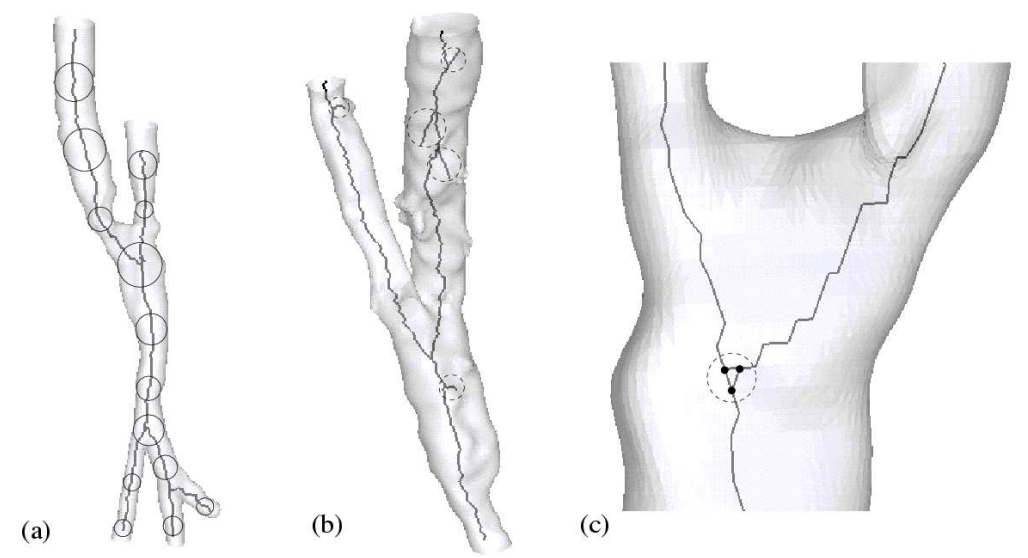

Figure 7: Skeletons from the thinning algorithm: (a) example of skeleton where the circles represent the maximum interior sphere with its centre at the vessel centerline; (b) a skeleton with spurious branches, indicated by the dashed circles, that requires pruning; (c) three points (encircled) are candidates to be the bifurcation point.

An alternative approach based on the smoothing of a triangulation representing the surface is proposed in [20]. The first step is to apply a Laplacian filter where the coordinates $\mathbf{x}_{i}$ of a vertex $i$ on the surface triangulation are moved to a new position

$$
\mathbf{x}_{i}^{*}=\mathbf{x}_{i}+\lambda \delta \mathbf{x}_{i}
$$

with $\lambda>0$ and

$$
\delta \mathbf{x}_{i}=\sum_{j=1}^{N} w_{i j}\left(\mathbf{x}_{j}-\mathbf{x}_{i}\right) .
$$

Here the summation extends over the $N$ vertices in the triangulation connected with the vertex $i$ by a side and the weights $w_{i j}$ are positive numbers that satisfy $\sum_{j=1}^{N} w_{i j}=1$. This smoothes the surface but also shrinks it. To compensate for this, an anti-smoothing step is applied where

$$
\mathbf{x}_{i}^{\prime}=\mathbf{x}_{i}^{*}+\mu \delta \mathbf{x}_{i}
$$

with $\delta \mathbf{x}_{i}$ given by equation (9) and $\mu<-\lambda$. The combination of these two steps leads to a low-pass filter that reduces high-order frequencies without shrinking the object. The results of this method applied to the model case are shown in figure $6(\mathrm{~d})$. This method can produce better results than the Gaussian convolution filter due to its non-shrinking properties but still requires a judicious selection of the weights $w_{i j}$ and the scale factors $\lambda$ and $\mu$.

\section{Centreline extraction}

The medial line of a complex three dimensional object is the locus of the centres of the maximal spheres inside the object. It is sometimes referred to as its skeleton. A sphere interior to an object is maximal when it is not contained in any other sphere interior to the object [7] as illustrated in figure $7(\mathrm{a})$. The geometrical description of a shape in terms of its skeleton is made up of the skeletal points, and the radius of the maximal sphere associated with each point.

Within this work, a fast, six sub-iterations, three dimensional thinning algorithm generates the skeletal points that approximate the medial line of a three dimensional object. No information about the maximal spheres is presently computed and used within this work. A different approach to the approximation of medial lines based on Voronoi diagrams, as described in [2], would also 


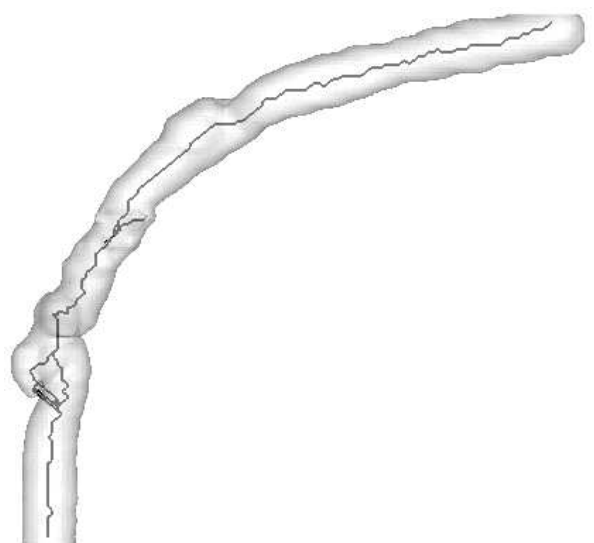

(a)

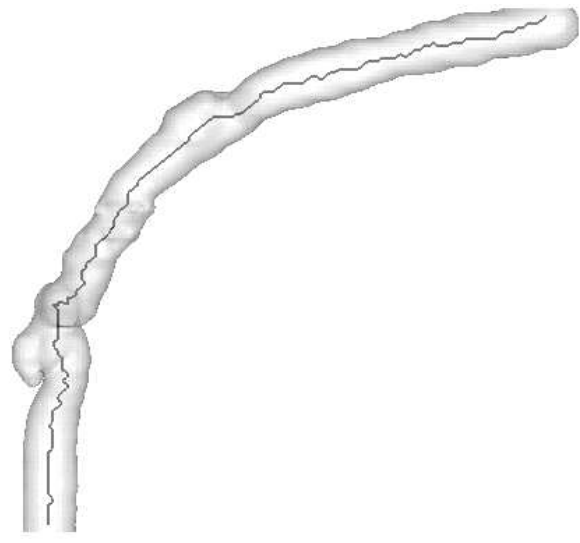

(b)

Figure 8: Pruning of a porcine carotid by-pass reconstruction: (a) original skeleton, (b) main vessel pruned by the Dijkstra shortest path algorithm.

determine the radii of the maximal spheres. The thinning algorithm is due to Palagyi and Kuba [15] and is implemented on a three dimensional binary image of a reconstructed anastomosis. This is generated by building a structured cube of three dimensional pixels (voxels) whose intensities can only be 0 or 1 . The intensity of a voxel is 0 if it is outside the object, i.e. it is part of the background of the picture, or 1 if it is inside. The algorithm works by iteratively removing those voxels that are on the boundary of the object, thinning it until only its skeleton (a single pixel) remains. An analysis of how accurately the algorithm approximates the medial line and how sensitive it is to perturbations of the surface of the object can be found in [15].

Since the thinning algorithm requires each voxel to know the status of its 26 neighbours, the skeleton that is produced at the end of the thinning is a set of points that preserves some connectivity information. So, it is possible to build a graph of the approximated medial line as depicted in figure 7 .

Further operations might be necessary to obtain an acceptable medial line as shown in figure 7(b). First, the roughness of the reconstructed surface may generate some branches that are not consistent with the main topology of the branching vessels. That is, by looking at the medial line only, one may identify some spurious branches that are not really present. Using a gardening analogy, the process of removing these branches is called pruning. The medial lines that result from the thinning algorithm usually require pruning. Finally, the graph derived from the skeletonisation can provide misleading connectivity information when trying to identify the bifurcation point of branching vessels. This is illustrated in figure $7(\mathrm{c})$ which shows three candidate points for the bifurcation point. Choosing the bifurcation point amongst any of the three options does not introduce any significant error since the resolution of the skeletonisation algorithm is higher than that of the MR images.

The strategy followed to perform the pruning is based on operations that can be performed on graphs. Specifically, the user manually selects the bifurcation point and identifies the ends of the medial lines of the three branches that originate from the bifurcation. The end of a branch is the point in the medial line which is the farthest from the bifurcation. The centreline of each branch is then defined as the shortest path from the bifurcation to the end. The single source shortest path algorithm proposed by Dijkstra [1] provides a consistent way to track a branch given its end points. It uses a weighted graph with weights proportional to the arc lengths of the branches forming the medial line. This algorithm consistently produces reasonable results even when starting from a complicated medial line structure as illustrated in figure 8 . 


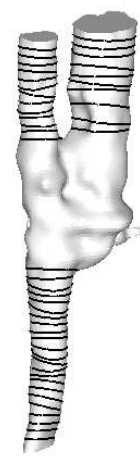

(a)

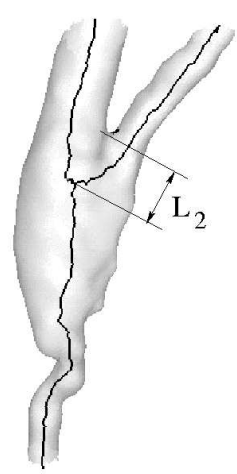

(b)

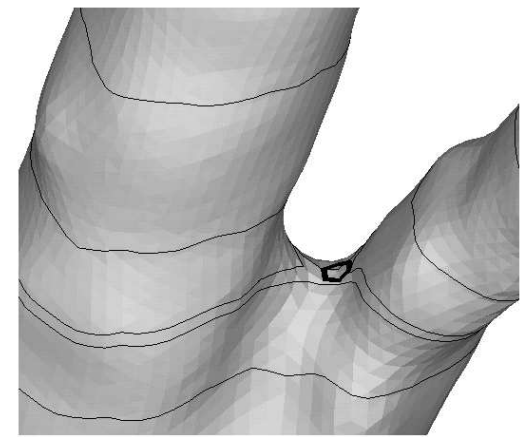

(c)

Figure 9: Determination of the cross-sectional area distribution along the medial line: (a) typically it is necessary to visually isolate the distal host vessel from the anastomosis region; (b) the distance between the apex and the junction is the reference length $\mathrm{L}_{2}$; (c) identification of an apex point as the vertex of a topological change, the apex is bounded by a thick black polygon.

\section{Cross-sectional area distribution along the medial line}

The distribution of cross-sectional area along the medial line of each branch of an anastomosis can be computed by slicing the triangulation obtained from the reconstruction with planes that are perpendicular to the medial line. The medial line is the result of the thinning of a binary image. It is therefore necessary to smooth this pixelised representation to obtain a suitable curve from which the normal to the slicing plane can be evaluated. We define the normal to the slicing plane as the average tangent vector to the medial line at each point, using at least 10 consecutive neighbours about the point of interest to perform the average.

Cross-sectional areas are computed only where each of the three vessels behaves like a tube. This leaves an arbitrary choice as to where the distal host vessel becomes an independent branch as opposed to being part of the junction. This question can be addressed visually while considering each individual case as highlighted in figure 9 (a).

The determination of a cross-sectional area distribution along all the available length of the data allows us to define a reference length as the mean diameter of the three vessels. After computing the mean cross-sectional areas of each branch $A_{G}, A_{P}$ and $A_{D}$, where the subscripts denote the graft $(\mathrm{G})$, and the proximal (P) and distal (D) branches of the host artery, the reference length $\mathrm{L}_{1}$ is taken to be the diameter of a circle with area equal to the average sectional area:

$$
\mathrm{L}_{1}=2 \sqrt{\frac{A_{G}+A_{D}+A_{P}}{3 \pi}} .
$$

Another reference length $\mathrm{L}_{2}$ that measures the magnitude of an anastomosis can be defined as the distance between the bifurcation point of the medial lines and a significant vertex on the triangulated surface (referred to as the apex). The apex can be identified using the procedure proposed by Ferley et al. [7]. This is a modification of Dijkstra's single source shortest path algorithm. Given a source vertex located at the end of the distal host vessel, this algorithm associates each vertex belonging to the triangulation to its shortest distance to the source. This distance is computed on the undirected graph obtained from the connectivity of the triangulation. The apex is the vertex where isocontours of the distance from the source vertex change their topology. Moving away from the source, isocontours of the distance from the source are closed and unique. Once the apex is reached, there are two closed isocontours of the same distance value as shown in figure $9(\mathrm{c})$.

If $D_{i}$ is the distance from the source to vertex $i$, and if the $i$ th vertex has $\mathrm{N}$ neighbours whose distance from the source is $D_{j}$ for $j=1, \ldots, \mathrm{N}$, counting the number of sign changes of the $\mathrm{N}$ 
quantities $D_{j}-D_{i}$ is sufficient to determine whether a topology change happens at the $i$ th vertex. Details of the algorithm are given in [7].

\section{Parameters for shape classification}

The skeletonisation and the shortest path algorithms provide the centrelines of the reconstructed blood vessels as a weighted graph. In the case of a bifurcation, three vessels start from a common point.

A range of parameters can be chosen to describe an anastomosis which could then be used to classify and group data from different patients. The same parameters may also be useful to relate the bulk flow features to salient aspects of the geometry. These parameters must therefore be representative of an anastomosis and also intuitively comprehensible in the sense that they should be easily visualisable when an anastomosis is performed in the operating theatre.

The dominant features that one can extract from the approximation of centrelines are angles which will therefore be our initial focus. Following the notation of figure 10, we define three angles. GPA denotes the angle between the graft and the proximal host vessel, GDA is the angle between the graft and the distal host vessel, and PDA represents the angle between the proximal and the distal host vessels.

As a first attempt, each vessel was approximated by a straight line using a least-square fit constrained to pass through the bifurcation point. This produced three lines starting from the bifurcation point whose direction changes according to how much information is conveyed into the approximation. An example of this approximation is shown in figure 11. Starting with a medial line approximation shown in figure 11(a), the centreline of each vessel was parametrised according to its arc length and non-dimensionalised by the reference length $\mathrm{L}_{1}$. Lines were then fitted using all the points whose arc length from the bifurcation was within integer multiples of $\mathrm{L}_{1}$. This yielded a set of lines for each anastomosis as shown in figure 11(b).

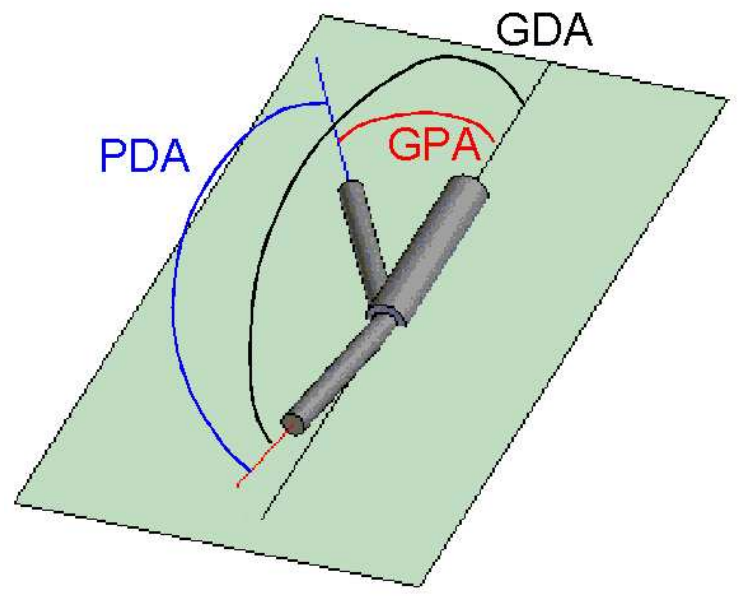

Figure 10: Definition of the angles between the graft and the proximal and distal host vessels: GPA is the angle between the graft and the proximal host vessel, GDA the angle between the graft and the distal host vessel, and PDA the angle between the proximal and the distal host vessels.

By computing the inner product of unitary vectors aligned to the least-squares linear fits of the graft, proximal host and distal host, we are able to measure the angles between the three vessels at the junction, GPA, GDA and PDA. Figure 11(d) shows the variation of the values of the three angles with the dimensions of the region of interest expressed as multiples of $\mathrm{L}_{1}$. At a distance of $3 L_{1}$ the angles were 66,134 and 158 for GPA, GDA and PDA respectively. 


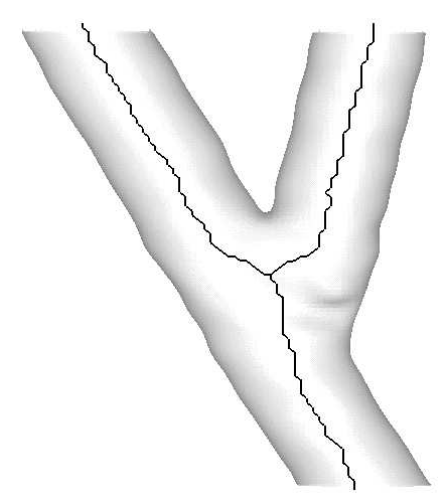

(a)

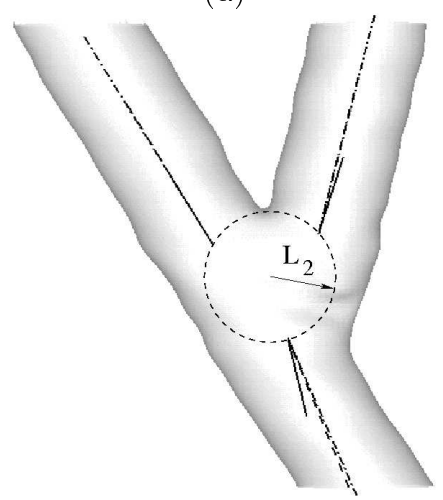

(c)

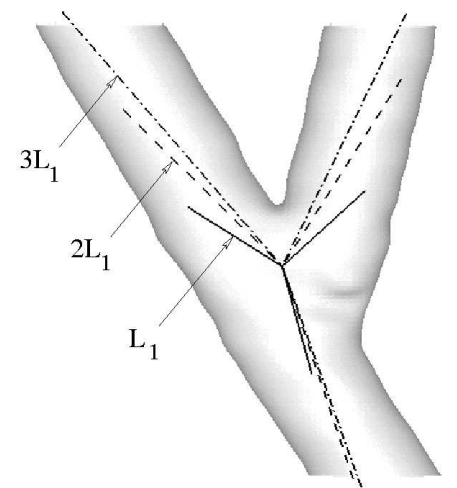

(b)

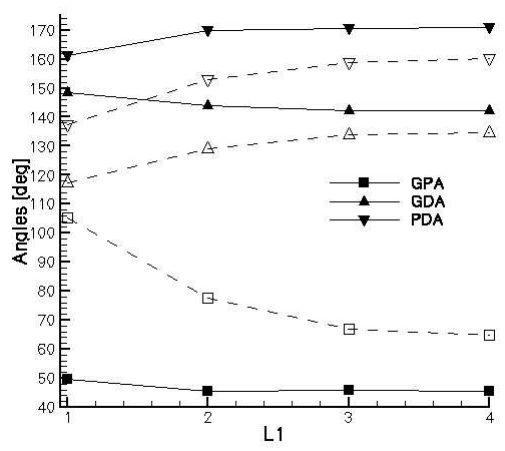

(d)

Figure 11: Centreline identification: (a) skeleton of a model anastomosis, (b) linear approximation to the skeleton using data from a sphere centred at the bifurcation point and of radius $-\mathrm{L}_{1}$, $---2 \mathrm{~L}_{1}$ and $-\cdot-3 \mathrm{~L}_{1} ;$ (c) linear approximation of points which are farther than $\mathrm{L}_{2}$ from the bifurcation, $(\mathrm{d})$ variation of the angles with inspection length in multiples of $\mathrm{L}_{1}$ (dashed lines refer to case (b), continuous lines refer to case (c)).

The analytic values of the three angles GPA, GDA and PDA based upon the vessel centrelines are 45, 135 and 180 degrees, respectively. Therefore we see that the angles computed by the linear approximation fixed to the bifurcation point as shown in figure 11(b) do not match the analytical results very well. The reason for this is that the position of the bifurcation point determined by the skeletonisation algorithm is at the centre of a maximal sphere and therefore cannot coincide with the point where the axes of the two cylinders intersect.

Nevertheless the definition of the reference length $\mathrm{L}_{2}$ provides an appropriate scale to help alleviate this problem. We recall that $\mathrm{L}_{2}$ characterises the anastomosis cuff and it is this region that we would like to ignore in our angle calculation. Therefore if we approximate the three branches by least-squares linear fits that are not constrained to go through the bifurcation point and exclude all points within a sphere of radius $\mathrm{L}_{2}$ centred at the bifurcation point, we obtain the approximations shown in figure 11(c).

The angles GPA, GDA and PDA were then computed by using the new lines and were found to be 45, 141 and 170 degrees respectively which is within $3 \%$ of the analytic values. As figure 11(d) also shows, disregarding the information inside the anastomosis region as defined by $\mathrm{L}_{2}$ reduces the variability of the measures.

A series of peripheral arterial bypass grafts were scanned with prior consent by the patients. The linear approximation, excluding the medial lines within the sphere of radius $\mathrm{L}_{2}$, was also applied to these anatomically correct reconstructions, yielding the results of table 1. 

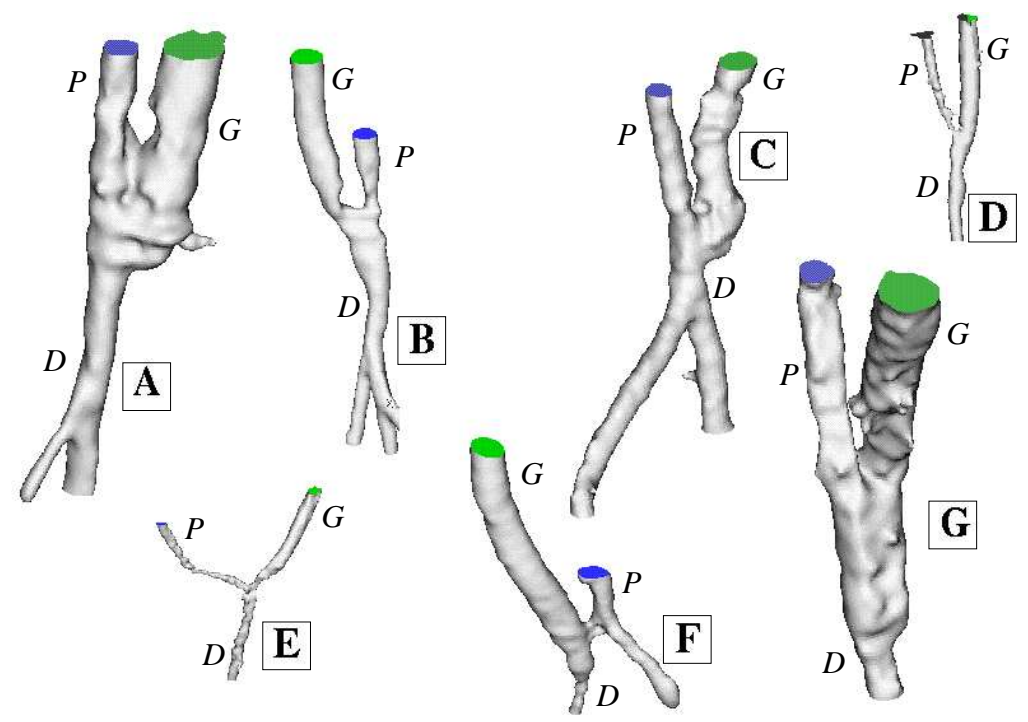

Figure 12: Reconstructions of the anastomoses described in table 1. The branches of the bifurcation are denoted as follows: Graft $(G)$, proximal host $(P)$, and distal host $(D)$.

\begin{tabular}{l|l|l|l|l|l} 
Case & GPA $[\mathrm{deg}]$ & GDA $[\mathrm{deg}]$ & PDA $[\mathrm{deg}]$ & $\mathrm{I}[\mathrm{deg}]$ & $\mathrm{L}_{2} / \mathrm{L}_{1}$ \\
\hline A & 9 & 176 & 172 & 3 & 1.6 \\
A2 & 9 & 168 & 177 & 7 & 1.6 \\
B & 26 & 150 & 171 & 1 & 1.3 \\
B2 & 24 & 157 & 172 & 1 & 1.4 \\
B3 & 39 & 138 & 176 & 2 & 1.2 \\
B4 & 43 & 142 & 167 & 1 & 1.0 \\
C & 8 & 169 & 163 & 1 & 1.0 \\
D & 35 & 168 & 153 & 7 & 1.3 \\
D2 & 32 & 177 & 150 & 7 & 1.2 \\
E & 97 & 143 & 119 & 15 & 0.6 \\
F & 50 & 155 & 152 & 16 & 0.9 \\
G & 26 & 152 & 172 & 30 & 1.4 \\
G2 & 26 & 156 & 176 & 30 & 1.1 \\
H & 27 & 157 & 136 & 6 & 1.5 \\
H2 & 30 & 165 & 137 & 1 & 1.4 \\
I & 42 & 150 & 155 & 28 & 0.5 \\
L & 31 & 141 & 166 & 13 & 2.2 \\
M & 39 & 156 & 164 & 3 & 1.1 \\
N & 80 & 128 & 148 & 32 & 0.8 \\
O & 13 & 155 & 163 & 13 & 0.8 \\
\hline MEAN & 34.3 & 155.1 & 159.5 & 10.9 & 1.19 \\
\hline SD & 22.1 & 12.9 & 15.7 & 10.9 & 0.39 \\
\hline
\end{tabular}

Table 1: Geometric classification for a set of in-vivo peripheral arterial bypass grafts. The surface reconstruction for cases $\mathrm{A}$ to $\mathrm{G}$ are depicted in figure 12, the number in the case designation indicates a multiple MRI exam. The values of GPA, GDA and PDA were evaluated over a region of interest of radius $4 \mathrm{~L}_{1}$. The last two rows contain the mean and the standard deviation of the data. 


\subsection{Follow-up of in-vivo geometry}

The reconstruction technique can also be applied when the same patient is re-scanned to check the patency of the graft. As indicated in table 1, Case B was scanned four times. The first three scans were taken one month, two months (Case B2) and three months (Case B3) respectively after surgery while the fourth scan (Case B4) was taken nine months after in a higher specification scanner. This scanner is a Siemens Sonata 1.5 Tesla with peak gradient strength of $40 \mathrm{mT} / \mathrm{m}$. The parameters for this scan are: field of view (FOV) $13 \mathrm{~cm}$, phase FOV 0.75, spatial resolution $512 \times 256$, TE $6 \mathrm{~ms}$, TR $31 \mathrm{~ms}$, and a slice thickness of $1 \mathrm{~mm}$.

The reconstruction of the anastomosis of this patient is shown in figure 13 that includes the first, second, third and fourth post-operative scans. Even though the angles of table 1 change only slightly, the reconstruction of Case B3 shows a noticeable narrowing of both the anastomosis cuff and the proximal host vessel, which is confirmed by the follow-up scan B4. The angle GPA measured from the fourth scan is greater than the others as a consequence of this narrowing. Since the fourth scan showed that the narrowing has stopped, no further operation was planned on this patient.

The results obtained from the different scanner used for Case B4 and shown in figure 13(d) provide some confidence in the robustness of the classification parameters with respect to the resolution of the MRI scanner. Furthermore this case also illustrates the sensitivity to smoothing. It seems that the patient has moved during the scan since the reconstructed anastomosis shows some undulations at the cuff. Even without smoothing the reconstruction to remove the undulations, the measured parameters are clearly in line with the previous scans.

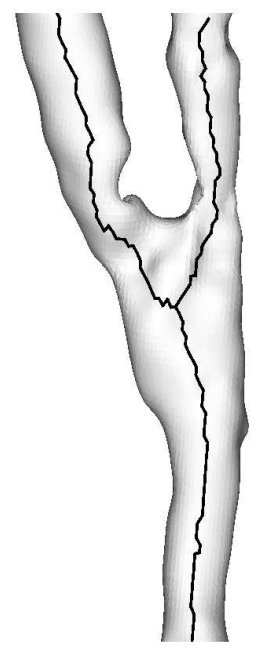

(a)

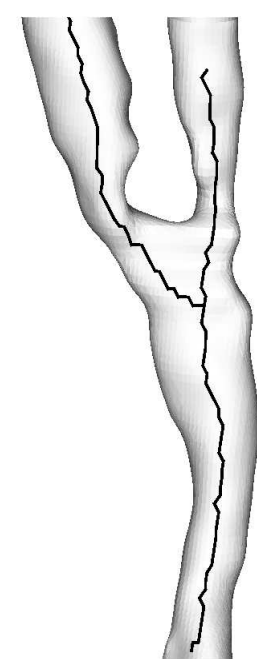

(b)

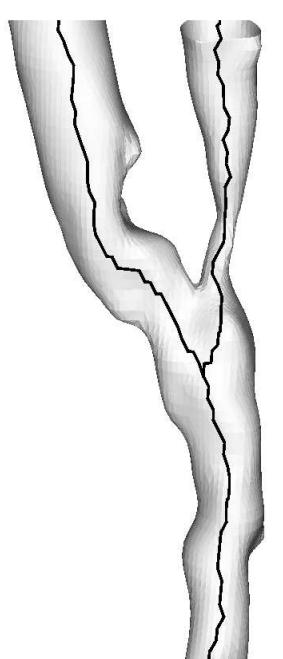

(c)

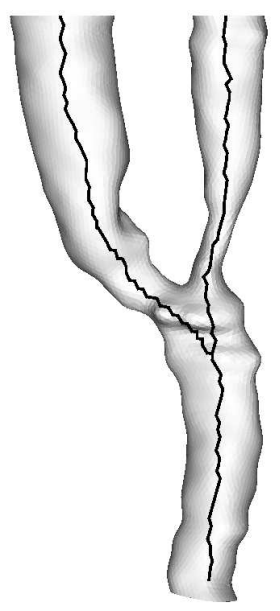

(d)

Figure 13: (a) Reconstruction of Case B scanned one month after the operation; (b) two months postoperatively (Case B2), showing just a slight reduction of the size of the anastomosis; (c) three months after the operation (Case B3) the anastomosis cuff has narrowed considerably, the three vessels have reduced their diameter and the proximal host vessel is developing a stenosis; (d) a scan performed nine months after the operation (Case B4) in a higher resolution scanner, confirms the evolution of the geometry and shows that the narrowing of the proximal host has apparently stopped.

\subsection{Definition of a reference plane}

The planarity of an anastomosis can have an important influence on blood flow [19], although the definition and the quantification of planarity of an in-vivo graft is not well established. As proposed 
in [10], planarity can be quantified by considering the singular value decomposition (SVD) of a matrix whose rows contain the coordinates of the points that belong to the medial line. Although this approach is mathematically well defined it is not so amenable to a visual interpretation and so is of limited application in a clinical environment.

In the case of peripheral by-pass grafts, most of the flow entering the anastomosis is carried through the grafted vessel. It may be important to know whether or not the graft lies in the same plane as the host vessel and the anastomosis cuff. A similar approach was followed by Friedman and Ding [8] with the aim of quantifying the planarity of the aortic bifurcation acquired from in-vivo MRI. These authors define an index of planarity based on the angle that the axis of the parent vessel forms with the plane that contains the axis of the two daughter vessels. Such a reference plane cannot be defined robustly when considering distal anastomoses of peripheral bypass grafts. In such cases the daughter vessels are actually just one artery. Therefore the proximal and distal part of the native host vessel may lie on a straight or almost straight line depending on inter-patient variability and on the surgical technique used while performing the anastomosis. This implies that the definition of a reference plane following Friedman and Ding [8] would be ill-conditioned as there is an infinite number of planes that contain a single line.

For each reconstructed anastomosis it is possible to define a reference plane as shown in figure 14 (a). This plane is a least squares fit to the points on the proximal and distal host vessels medial line that are within $3 \mathrm{~L}_{1}$ of the bifurcation point and the points on the graft medial line that are within $\mathrm{L}_{2}$ from the bifurcation point.

This reference plane will only contain the graft when its medial line is co-planar outside the anastomosis cuff. We note that the choice of $3 \mathrm{~L}_{1}$ is relatively arbitrary and we could have limited the definition to the anastomosis region by choosing $\mathrm{L}_{2}$ in all directions. However, using the larger value $3 \mathrm{~L}_{1}$ makes the reference plane less sensitive to the local noise within the host vessel, especially as $\mathrm{L}_{2}$ can be quite small for some data. The angle, I, between the straight line approximating the medial line of the graft and the reference plane can be used as a measure of the planarity of the configuration.

It can be appreciated that the angle I is easily visualisable and so has the potential of being evaluated in the surgical environment. An illustration of the reference plane and angle I is shown in figure 14. For the set of in-vivo data acquired in this study (see figure 12) the angles I are given table 1.

\section{Conclusions}

The work presented in this paper consists of the development and the application of computer visualisation tools to the reconstruction and the systematic characterisation of the distal anastomosis of peripheral by-pass grafts from data acquired in-vivo using MRI. The proposed techniques are highly automated and require a minimum of user intervention during the process of geometry reconstruction and characterisation. The availability of automated tools has two advantages. First, it reduces the user intervention and thus increases the speed of the process. This is highly desirable when dealing with a large number of patients. Second, keeping the user-defined parameters to a minimum reduces the subjectivity of both the reconstruction and the characterisation, aiding to the reproducibility of the results.

A preliminary analysis of the data in table 1 shows that the distal anastomoses of peripheral by-pass grafts possess quite similar values of the two angles GDA and PDA. This cannot be said of the angle GPA. The available data base, although limited, shows that the angle GPA allows the definition of two families of anastomoses. One of the families contains all the anastomoses such as cases A and B, where GPA is "shallow". The other family contains those anastomoses like case $\mathrm{E}$ and N, whose GPA is much larger (even greater than 90 degrees). The need to introduce a distinction between these two families seems to be confirmed by the much higher standard deviation of GPA when compared with the standard deviation of GDA and PDA.

The standard deviation of the angle I is equal to the mean value. This implies that there must be some distinction between planar and non-planar configurations. Planar anastomoses are those 
such as in figure 14(b), whose angle I is small (i.e. less than 10 or 15 degrees). The standard deviation of I reduces to 4.7 degrees when non-planar configurations (I greater than 15 degrees) are considered separately from the rest.

To conclude, the current data set of reconstructed geometries suggests that the apparently vast spectrum of anastomoses may be reduced to a relatively small subset of cases in terms of the non-planar angle I and the angle GPA. Furthermore, there is a relation between the angle GPA and the surgical technique adopted to construct the by-pass graft [5]. Grafts that are realised by tunnelling the vein deep inside the leg show a lower GPA (mean GPA $=26$ degrees) as opposed to those whose vein runs more superficially (mean GPA $=53$ degrees).

A number of issues still need to be addressed in future work. First, the global accuracy of the reconstruction method, including the fidelity of the scans, needs to be assessed. As far as the extraction of the medial line is concerned, small perturbations of the reconstructed surface do not significantly affect the skeletonisation algorithm, and therefore barely affect the computation of the angles and the reference plane, since these are least-squares approximations of the medial line. This is supported by the angle classification of the model anastomosis in figure 6 before and after smoothing where only a $3 \%$ variation was observed. Smoothing of the reconstructed surfaces may be required before generating a CFD mesh and it can be accomplished either by using the Gaussian smoothing or by applying the non-shrinking smoothing algorithm described in this paper and proposed in [20].

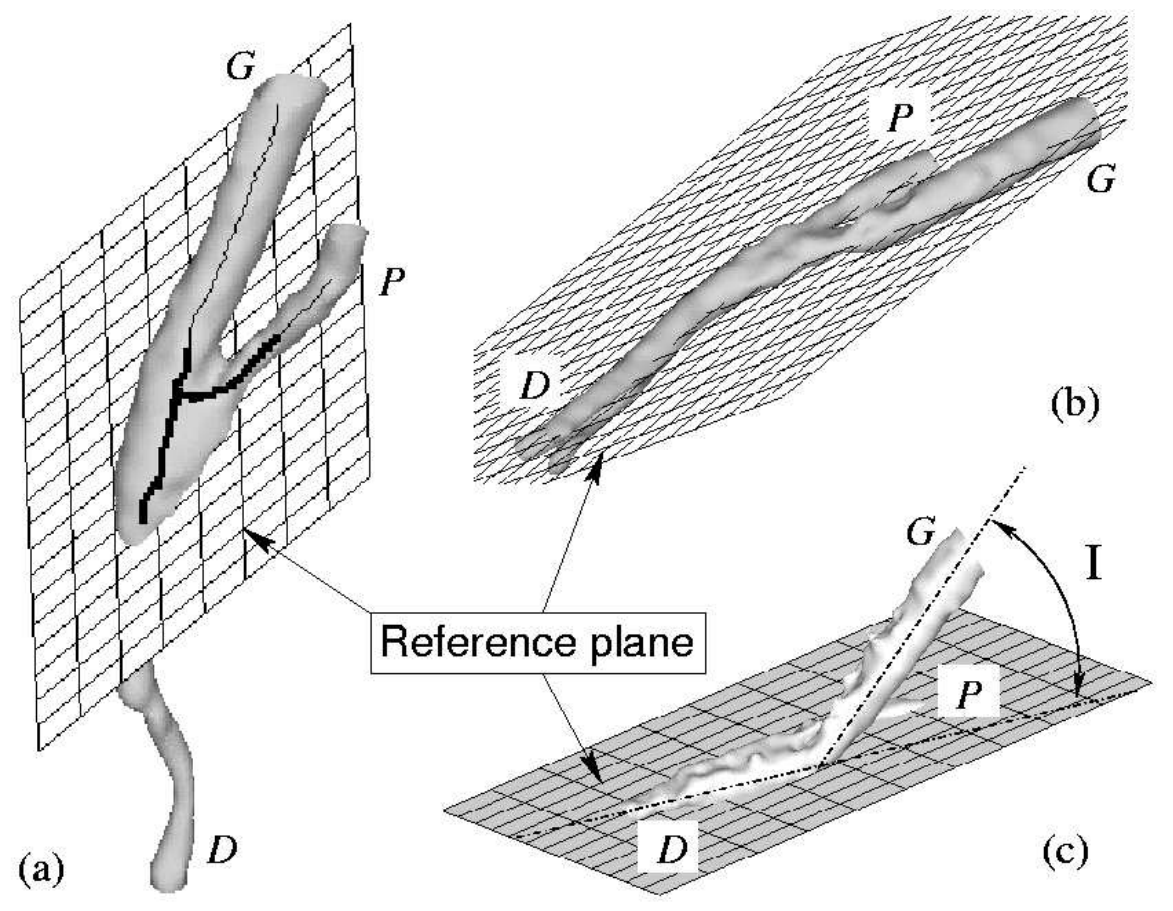

Figure 14: (a) Anastomosis reference plane. The points on the medial line that it approximates in a least-squares sense are highlighted as a thicker line. (b) In a "planar case" the graft lies almost completely on the reference plane. (c) In a "non-planar" case, the graft meets the reference plane at an angle I.

A further outstanding issue is the assessment of the relevance of the proposed parameters. Although the broad geometric features can be reduced to a small subset, these parameters may not be sufficient to fully capture the main haemodynamic features. This means that any classification solely based on shape will need to be reviewed to take into account the effects that shape has on the flow. At the same time, the parameter space may be enlarged to take into account the 
distribution of cross-sectional areas along the medial line. Such a quantification would also permit the patient-specific post-operative monitoring of the progress of a stenosis.

\section{Acknowledgements}

We are grateful for the support of the Henry Smith's Kensington Estate Charity, the Clothworkers' Foundation and the Garfield Weston Foundation.

We would also like to thank Dr Wadi Gedroyc, Paul Wragg, Rupi Gillard and Lisa Anderton of the MR Unit, St Mary's Hospital, for their assistance with the MR measurements on the patients.

\section{References}

[1] A. Aho. Data structures and algorithms. Addison-Wesley, 1983.

[2] D. Attali and A. Montanvert. Computing and simplifying 2D and 3D continuous skeletons. Comput. Vis. Image Understand., 67:261-273, 1997.

[3] J. Bloomenthal. An implicit surface polygonizer. In P. S. Heckbert, editor, Graphics Gems $I V$, pages 324-349. Academic Press, 1994.

[4] J. R. Cebral and R. Löhner. From medical images to anatomically accurate finite element grids. Int. J. for Num. Meth. in Engineering, 51:985-1008, 2001.

[5] J. S. Crane, M. J. Jackson, C. D. Bicknell, S. Giordana, J. Peiró, D. J. Doorly, S. J. Sherwin, N. J. W. Cheshire, and C. G. Caro. In-vivo geometric features of distal vein graft anastomoses: Magnetic resonance surface reconstruction in different graft techniques. Manchester, May 7-9 2003. Association of Surgeons of Great Britain and Ireland.

[6] J. Duchon. Spline minimizing rotation-invariant semi-norms in Sobolev spaces. In W. Schempp and K. Zeller, editors, Constructive Theory of Functions on Several Variables, number 571 in Lecture Notes in Mathematics, pages 85-99. Springer-Verlag, Berlin, 1977.

[7] E. Ferley, M. P. Cani-Gascuel, and D. Attali. Skeletal reconstruction of branching shapes. Computer Graphics Forum, 16(5):283-293, 1997.

[8] M. H. Friedman and Z. Ding. Variability of the planarity of the human carotid bifurcation. Medical Engineering and Physics, 20:469-472, 1998.

[9] G. E. Karniadakis and S. J. Sherwin. Spectral/hp element methods for CFD. Oxford University Press, 1999.

[10] E. King, X. Y. Xu, A. D. Hughes, Q. Long, S. A. Thom, and K. H. Parker. Quantification of the non-planarity of the human carotid bifurcation. In Breaking Symmetry in Haemodynamics, London, 2001. Imperial College.

[11] A. Leuprecht, K. Perktold, M. Prosi, T. Berk, W. Trubel, and H. Schima. Numerical study of hemodynamics and wall mechanics in distal end-to-side anastomoses of bypass grafts. Journal of Biomechanics, 35:225-236, 2002.

[12] Q. Long, X. Y. Xu, M. W. Collins, M. Bourne, and T. M. Griffith. Magnetic resonance image processing and structured grid generation of a human abdominal bifurcation. Computer Methods and Programs in Biomedicine, 56:249-259, 1998.

[13] J. A. Moore, D. A. Steinman, and C. R. Ethier. Computational blood flow modelling: errors associated with reconstructing finite element models from magnetic resonance images. Journal of Biomechanics, 31:179-184, 1998. 
[14] J. A. Moore, D. A. Steinman, D. W. Holdsworth, and C. R. Ethier. Accuracy of computational hemodynamics in complex arterial geometries reconstructed from magnetic resonance imaging. Annals of Biomedical Engineering, 27:32-41, 1999.

[15] K. Palagyi and A. Kuba. A 3D 6-subiteration thinning algorithm for extracting medial lines. Pattern Recognition Letters, 19(7):537-641, May 1998.

[16] J. Peiró, S. Giordana, C. Griffith, and S.J. Sherwin. High-order algorithms for vascular flow modelling. Int. J. for Numer. Meth. Fluids, 40, 2002.

[17] K. Perktold, M. Hofer, G. Rappitsch, M. Loew, K. D. Kuban, and M. H. Friedman. Validated computation of physiological flow in a realistic coronary artery branch. Journal of Biomechanics, 31:217-228, 1998.

[18] S. J. Sherwin and J. Peiró. Mesh generation in curvilinear domains using high-order elements. Int. J. Numer. Meth. Engng., 53:207-223, 2002.

[19] S.J. Sherwin, O. Shah, D.J. Doorly, J. Peiró, Y. Papaharilaou, N. Watkins, C.G. Caro, and C.L. Dumoulin. The influence of out-of-plane geometry on the flow within a distal end-to-side anastomosis. ASME Journal of Biomechanical Engineering, 122:1-10, 2000.

[20] G. Taubin. A signal processing approach to fair surface design. Computer Graphics, 29:351358, 1995. Annual Conference Series.

[21] G. Turk and J. F. O'Brien. Shape transformation using variational implicit surfaces. In Computer Graphics Proceedings, Annual Conference Series, SIGGRAPH99, pages 335-342, Los Angeles, CA, USA, August 8-13 1999. ACM. 